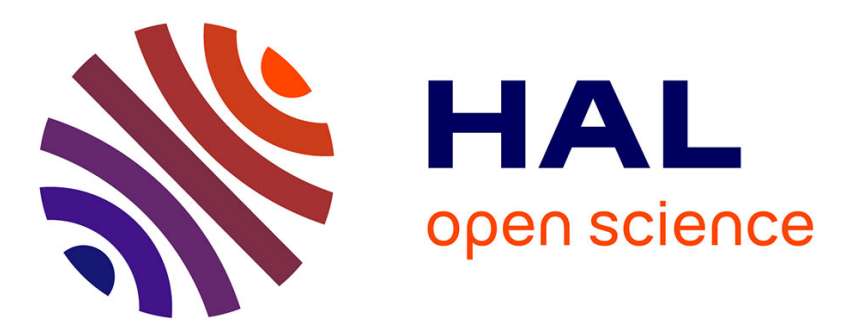

\title{
Bearing capacity of strip footings on spatially random soils using sparse polynomial chaos expansion
}

\author{
Tamara Al-Bittar, Abdul-Hamid Soubra
}

\section{To cite this version:}

Tamara Al-Bittar, Abdul-Hamid Soubra. Bearing capacity of strip footings on spatially random soils using sparse polynomial chaos expansion. International Journal for Numerical and Analytical Methods in Geomechanics, 2013, 37 (13), pp.2039-2060. 10.1002/nag.2120 . hal-01005286

\section{HAL Id: hal-01005286 \\ https://hal.science/hal-01005286}

Submitted on 5 Nov 2017

HAL is a multi-disciplinary open access archive for the deposit and dissemination of scientific research documents, whether they are published or not. The documents may come from teaching and research institutions in France or abroad, or from public or private research centers.
L'archive ouverte pluridisciplinaire HAL, est destinée au dépôt et à la diffusion de documents scientifiques de niveau recherche, publiés ou non, émanant des établissements d'enseignement et de recherche français ou étrangers, des laboratoires publics ou privés. 


\title{
Bearing capacity of strip footings on spatially random soils using sparse polynomial chaos expansion
}

\author{
Tamara Al-Bittar*, ${ }^{\dagger}$ and Abdul-Hamid Soubra \\ LUNAM Université, University of Nantes, GeM, UMR CNRS 6183, Bd. de l'université, BP 152, 44603 Saint-Nazaire \\ Cedex, France
}

\begin{abstract}
A probabilistic model is presented to compute the probability density function (PDF) of the ultimate bearing capacity of a strip footing resting on a spatially varying soil. The soil cohesion and friction angle were considered as two anisotropic cross-correlated non-Gaussian random fields. The deterministic model was based on numerical simulations. An efficient uncertainty propagation methodology that makes use of a non-intrusive approach to build up a sparse polynomial chaos expansion for the system response was employed. The probabilistic numerical results were presented in the case of a weightless soil. Sobol indices have shown that the variability of the ultimate bearing capacity is mainly due to the soil cohesion. An increase in the coefficient of variation of a soil parameter $(c \operatorname{or} \varphi)$ increases its Sobol index, this increase being more significant for the friction angle. The negative correlation between the soil shear strength parameters decreases the response variability. The variability of the ultimate bearing capacity increases with the increase in the coefficients of variation of the random fields, the increase being more significant for the cohesion parameter. The decrease in the autocorrelation distances may lead to a smaller variability of the ultimate bearing capacity. Finally, the probabilistic mean value of the ultimate bearing capacity presents a minimum. This minimum is obtained in the isotropic case when the autocorrelation distance is nearly equal to the footing breadth. However, for the anisotropic case, this minimum is obtained at a given value of the ratio between the horizontal and vertical autocorrelation distances.
\end{abstract}

KEY WORDS: bearing capacity; probabilistic analysis; spatial variability; sparse polynomial chaos expansion

\section{INTRODUCTION}

The spatial variability of the soil properties affects the behavior of geotechnical structures (bearing capacity, foundation settlement, slope stability, etc.). Several authors have considered the effect of the spatial variability of the soil properties in their calculation models. For the bearing capacity of foundations, which is the subject of the present paper, one may cite, among others, Griffiths and Fenton [1], Griffiths et al. [2], Fenton and Griffiths [3], Popescu et al. [4], Youssef Abdel Massih [5], Soubra et al. [6], Soubra and Youssef Abdel Massih [7] and Cho and Park [8].

It should be mentioned that when dealing with probabilistic studies that involve spatially varying soil properties, the classical Monte Carlo Simulation (MCS) methodology is generally used to determine the probability density function (PDF) of the system response. It is well known that this method is a very time-expensive approach. This is because of the following: (i) it generally makes use of finite element or finite difference models, which are generally time expensive; and (ii) it requires a great number of calls of the deterministic model. To overcome the inconvenience of the time cost, this paper presents

*Correspondence to: Tamara Al-Bittar, University of Nantes, Bd. de l'université, BP 152, 44603 Saint-Nazaire cedex, France.

'E-mail: Tamara.Al-Bittar@univ-nantes.fr 
a more efficient probabilistic approach, which significantly reduces the number of calls of the deterministic model. The sparse polynomial chaos expansion (SPCE) methodology was proposed in this regard. Notice that the sparse polynomial chaos expansion is an extension of the polynomial chaos expansion (PCE). A PCE methodology aims at replacing the deterministic model, which may be an analytical model or a finite element/finite difference model by a meta-model. This allows one to calculate the system response using a simple analytical equation (e.g. Isukapalli et al. [9]; Huang et al. [10]; Li et al. [11]; Mollon et al. [12]; Houmadi et al. [13]). This simple analytical equation is obtained by expanding the system response on a suitable basis, which is a series of multivariate polynomials that are orthogonal with respect to the joint probability density function of the input random variables. Consequently, the characterization of the PDF of the system response is equivalent to the evaluation of the PCE coefficients as it will be shown later in this paper.

The original PCE method is an intrusive approach in the sense that it requires extensive modifications in the deterministic code. In particular, the so-called spectral stochastic finite element method (SSFEM) was presented in [14] for mechanical problems involving random fields. In this approach, the classical finite element discretization is combined with the Karhunen-Loève decomposition of the input random fields. Then, the coefficients of the system response (i.e. the coefficients of the PCE) were obtained using a Galerkin scheme [15]. As an alternative, non-intrusive computational schemes emerged recently in stochastic finite element analysis. These methods allow one to compute the stochastic model response (i.e. the coefficients of the PCE) using a set of calls to the existing deterministic model (i.e. without modifying the underlying computer code). Two methods exist in literature for the computation of the PCE coefficients in the framework of the non-intrusive approaches: the projection method (e.g. Le Maitre et al. [16]) and the regression method (i.e. [9-13, 17, 18]).

Within the framework of the PCE, the PDF of the system response can be easily obtained. This is because MCS is no longer applied on the original expensive deterministic model but on the metamodel. This consists in performing a great number of realizations on the meta-model. It should be emphasized here that the obtained PCE coefficients can be used to perform a global sensitivity analysis based on Sobol indices. These indices give the contribution of each random field in the variability of the system response.

It should be noticed that the number of the PCE coefficients to be computed grows dramatically with the size of the input random vector and the PCE order. When dealing with random fields as is the case in the present paper, the discretization may lead to a significant number of random variables, which makes the solution of the PCE unfeasible. This is because of the proliferation of the unknown PCE coefficients to be computed. To address such problem, the sparse polynomial chaos expansion developed by Blatman and Sudret [18] in the framework of the non-intrusive approaches is used herein. This method is based on an adaptive regression-based algorithm, which automatically detects the significant coefficients of the PCE to be computed. As a consequence, a rather small number of the PCE coefficients is eventually retained (sparse representation), which may be obtained at a reduced computational cost compared with the classical 'full' PCE representation. It should be emphasized here that SPCE was applied by [18] to deal with problems where a large number of uncertain parameters were modeled as random variables. In this paper, the SPCE is applied to a two-dimensional (2D) spatially varying soil involving two random fields discretized into a great number of random variables (between 10 and 88 random variables depending on the values of the autocorrelation distances). More precisely, the aim of this paper is to compute the PDF of the ultimate bearing capacity of a shallow strip foundation resting on a 2D spatially varying $(c, \varphi)$ weightless soil. Although a ponderable soil can be used, the soil weight was neglected in this paper. This assumption was adopted to validate the present probabilistic approach with the use of a non-expensive deterministic model that has a reasonable computation time. Thus, the present analysis only provides the component of the ultimate bearing capacity related to the soil cohesion.

The soil cohesion and friction angle are considered as anisotropic cross-correlated non-Gaussian random fields. They are characterized by two specified marginal distribution functions, a common autocorrelation function, and a cross-correlation matrix. The methodology proposed by Vořechovsky [19] is used to generate these two random fields. The deterministic model is based on numerical simulations using FLAC ${ }^{3 \mathrm{D}}$ software. The adaptive algorithm to build up a SPCE is used to obtain an approximation of the analytical expression of the system response (ultimate bearing capacity). The 
paper is organized as follows: The next section aims at presenting the probabilistic method used for the computation of the PDF of the ultimate bearing capacity of a strip footing resting on a spatially varying soil. It is followed by the numerical results. The paper ends with a conclusion.

\section{PROBABILISTIC METHOD USED IN THE ANALYSIS}

First, the generation of two anisotropic cross-correlated non-Gaussian random fields is briefly presented. It is followed by a presentation of the probabilistic method adopted in this paper. Although the SPCE methodology is used for the probabilistic analysis, the PCE (based on the classical truncation scheme) is first presented, and then, it is followed by the presentation of the SPCE (based on the hyperbolic truncation scheme) to facilitate the understanding of the used approach. This section ends with a brief presentation of the post-treatment, which involves the following: (i) the statistical analysis of the system output; and (ii) the method of computation of the global sensitivity indices.

\subsection{Generation of anisotropic cross-correlated non-Gaussian random fields}

Let us consider two anisotropic cross-correlated non-Gaussian random fields $Z_{i}^{N G}(x, y)(i=c, \varphi)$ described by the following: (i) constant means and standard deviations $\left(\mu_{i}, \sigma_{i} ; i=c, \varphi\right)$; (ii) nonGaussian marginal cumulative density functions $G_{i}(i=c, \varphi)$; (iii) a target cross-correlation matrix $C^{N G}=\left(\begin{array}{cc}r_{c, c} & r_{c, \varphi} \\ r_{\varphi, c} & r_{\varphi, \varphi}\end{array}\right)$; and (iv) a common autocorrelation function $\rho_{Z}^{N G}\left[(\mathrm{x}, \mathrm{y}),\left(\mathrm{x}^{\prime}, \mathrm{y}^{\prime}\right)\right]$, which gives the values of the correlation function between two arbitrary points $(\mathrm{x}, \mathrm{y})$ and $\left(\mathrm{x}^{\prime}, \mathrm{y}^{\prime}\right)$. Notice that the exponential form of the autocorrelation function is the one that is the most commonly used in geotechnical engineering [4]. It is given as follows:

$$
\rho_{\mathrm{z}}^{N G}\left[(x, y),\left(x^{\prime}, y^{\prime}\right)\right]=\exp \left(-\left(\frac{\left|x-x^{\prime}\right|}{a_{x}}\right)^{n}-\left(\frac{\left|y-y^{\prime}\right|}{a_{y}}\right)^{n}\right)
$$

where $a_{x}$ and $a_{y}$ are the autocorrelation distances along $x$ and $y$ respectively. For $\mathrm{n}=1$, the autocorrelation function is said to be exponential of order 1 ; however, for $n=2$, it is said to be square exponential. In our study, we deal with two random fields (cohesion $c$ and friction angle $\varphi$ ) that are assumed to share an identical 2D square exponential autocorrelation function over the physical domain $\Omega$. The expansion optimal linear estimation method (EOLE) (proposed by Li and Der kiureghian [20] for the case of uncorrelated Gaussian random fields) and its extension by Vořechovsky [19] to cover the case of crosscorrelated non-Gaussian random fields are used herein to discretize the two random fields of $c$ and $\varphi$. In this method, one should first define a stochastic grid composed of $q$ grid points (or nodes) obtained from the different combinations of $H$ points in the $x$ (or horizontal) direction, and $V$ points in the $y$ (or vertical) direction assembled in a vector $Q=\left\{Q_{n}=\left(x_{h}, y_{v}\right)\right\}$ where $h=1, \ldots, H ; v=1, \ldots, V ; n=1, \ldots$, $q$ and $q=H x V$. Notice that for the vector $Q$ composed of $q$ elements, the values of the field are assembled in a vector $\chi=\left\{\chi_{n}=Z\left(x_{h}, y_{v}\right)\right\}$ where $h=1, \ldots, H ; v=1, \ldots, V$ and $n=1, \ldots, q$. Then, one should determine the correlation matrix, for which each element $\left(\Sigma_{\alpha ; i}^{N G}\right)_{i, j}$ is calculated using Equation (1) as follows:

$$
\left(\Sigma_{\gamma: \varkappa}^{N G}\right)_{i, j}=\rho_{z}^{N G}\left[Q_{i}, Q_{j}\right]
$$

where $i=1, \ldots, q$ and $j=1, \ldots, q$. Notice that the matrix $\Sigma_{\gamma ; \chi}^{N G}$ in Equation (2) provides the correlation between each element in the vector $\chi$ and all the other elements of the same vector. The common nonGaussian autocorrelation matrix $\Sigma_{\chi ; \chi}^{N G}$ and the target non-Gaussian cross-correlation matrix $\mathrm{C}^{\mathrm{NG}}$ should be transformed into the Gaussian space using the Nataf correction functions proposed by Nataf [21] because the discretization of the random fields using EOLE is carried out in the Gaussian space. This can be performed by applying the following formulas: 


$$
\begin{array}{cc}
\left(\Sigma_{\chi ; \chi}^{k}\right)_{i, j}=\omega_{i, j}\left(\Sigma_{\chi ; \chi}^{N G}\right)_{i, j} ; & i=1, \ldots q ; j=1, \ldots q \text { and } k=c, \varphi \\
C_{i, j}=\omega_{i, j} C_{i, j}^{N G} ; & i=c, \varphi \text { and } j=c, \varphi
\end{array}
$$

where $\omega_{i, j}$ is the correction factor. As a result, one obtains two Gaussian autocorrelation matrices $\Sigma_{\chi ; \chi}^{c}$ and $\Sigma_{\chi ; \chi}^{\varphi}$ and a Gaussian cross-correlation matrix $C$ that can be used to discretize the two random fields using EOLE as follows:

$$
\begin{gathered}
\tilde{Z}_{c}(x, y)=\mu_{c}+\sigma_{c} \sum_{j=1}^{N} \frac{\kappa_{c, j}^{D}}{\sqrt{\lambda_{j}^{c}}} \cdot\left(\phi_{j}^{c}\right)^{T} \cdot \Sigma_{Z(x, y) ; \chi}^{c} \\
\tilde{Z}_{\varphi}(x, y)=\mu_{\varphi}+\sigma_{\varphi} \sum_{j=1}^{N} \frac{\kappa_{\varphi, j}^{D}}{\sqrt{\lambda_{j}^{\varphi}}} \cdot\left(\phi_{j}^{\varphi}\right)^{T} \cdot \Sigma_{Z(x, y) ; \chi}^{\varphi}
\end{gathered}
$$

where $\left(\lambda_{j}^{c}, \phi_{j}^{c}\right)$ and $\left(\lambda_{j}^{\varphi}, \phi_{j}^{\varphi}\right)$ are the eigenvalues and eigenvectors of the two Gaussian autocorrelation matrices $\Sigma_{\chi ; \chi}^{c}$ and $\Sigma_{\chi ; \chi}^{\varphi}$, respectively; $\Sigma_{Z(x, y) ; \chi}$ is the correlation vector between the random vector $\chi$ and the value of the field at an arbitrary point $(x, y)$ as obtained using Equation (1), and finally $N$ is the number of terms (expansion order) retained in the EOLE method. This number will be determined later in this section. Notice that $\left(\phi_{j}\right)^{T}$ and $\Sigma_{Z(x, y) ; \chi}$ in Equations (5) and (6) are two vectors of dimensions (1x $q)$ and ( $q \times 1)$, respectively, and the superscript ' $T$ ' in $\left(\phi_{j}\right)^{T}$ denotes the transpose of the vector $\phi_{j}$. Notice finally that $\left(\kappa_{c, j}^{D}, \kappa_{\varphi, j}^{D}\right)$ are two cross-correlated blocks of independent standard normal random variables obtained using the Gaussian cross-correlation matrix $C$ between the two fields as follows: (i) one should compute the diagonal eigenvalues matrix $\Lambda^{C}$ with its corresponding eigenvectors matrix $\Phi^{C}$ of the Gaussian cross-correlation matrix $C$ using the spectral decomposition of the cross-correlation matrix $C$; and (ii) generate the block sample vector $\kappa^{D}$, which contains the two cross-correlated blocks $\left(\kappa_{c, j}^{D}, \kappa_{\varphi, j}^{D}\right)$ of independent standard random variables using the following formula:

$$
\kappa^{D}=\Phi^{D}\left(\Lambda^{D}\right)^{1 / 2} \xi^{T}
$$

where $\Phi^{D}$ is a $(2 N \mathrm{x} 2 N)$ block matrix resulting from the multiplication of each element in the matrix $\Phi^{C}$ by the unit matrix of order $N$ (the expansion order), $\Lambda^{D}$ is a $(2 N \mathrm{x} 2 N)$ block matrix resulting from the multiplication of each element in the matrix $\Lambda^{C}$ by the unit matrix of order $N$ and $\xi=$ $\left\{\xi_{c}=\left(\xi_{1}^{c}, \ldots, \xi_{N}^{c}\right), \xi_{\varphi}=\left(\xi_{1}^{\varphi}, \ldots, \xi_{N}^{\varphi}\right)\right\}$ is a block vector, which contains two blocks $\left(\xi_{c}, \xi_{\varphi}\right)$ of $N$ standard Gaussian independent random variables for each one.

Once the two Gaussian cross-correlated random fields are obtained, they should be transformed to the non-Gaussian space by applying the following formula:

$$
\tilde{Z}_{i}^{N G}(x, y)=G_{i}^{-1}\left\{\Phi\left[\tilde{Z}_{i}(x, y)\right]\right\} \quad i=c, \varphi
$$

where $\Phi($.$) is the standard normal cumulative density function. It should be mentioned here that the series$ given by Equations (5) and (6) are truncated for a number of terms $N$ (expansion order) smaller than the number of grid points $q$, after sorting the eigenvalues $\lambda_{j}^{c}$ and $\lambda_{j}^{\varphi}(j=1, \ldots, N)$ in a descending order. This number should assure that the variance of the error is smaller than a prescribed tolerance $\varepsilon$. Notice that the variance of the error for EOLE is given by [20]: 


$$
\operatorname{Var}\left[Z_{i}(x, y)-\tilde{Z}_{i}(x, y)\right]=\sigma_{Z}^{2}-\sum_{j=1}^{N} \frac{1}{\lambda_{j}^{i}}\left(\left(\phi_{j}^{i}\right)^{T} \Sigma_{Z(x, y) ; \chi}\right)^{2}(i=c, \varphi)
$$

where $Z_{i}(x, y)$ and $\tilde{Z}_{i}(x, y)$ are, respectively, the exact and the approximate values of the random fields at a given point $(x, y)$ and $\left(\phi_{j}^{i}\right)^{T}$ is the transpose of the eigenvector $\phi_{j}^{i}$ where $i=c, \varphi$.

\subsection{Polynomial chaos expansion-the classical truncation scheme}

The polynomial chaos expansion or its extension (sparse polynomial chaos expansion) aims to replace a complex deterministic model (i.e. finite element/finite difference numerical model) by a meta-model. This allows one to calculate the system response using an approximate simple analytical equation [9-13, 17, 18, 22-26]. Thus, the PCE or the SPCE may be used to perform a probabilistic analysis with a significant reduction in the computation time. This section is devoted to the PCE methodology.

The PCE makes use of multivariate polynomials, which are orthogonal with respect to the joint probability density function of the input random vector. The different types of the joint probability density functions and their corresponding multivariate polynomials are given by [23]. The Gaussian joint probability density function and its corresponding Hermite multivariate polynomials are used in this paper. Notice that the coefficients of the PCE may be efficiently computed using a non-intrusive technique where the deterministic calculations are carried out using, for example, a finite element or finite difference software treated as a black box. The most used non-intrusive method is the regression approach [9-13, 17, 18, 24-26]. The PCE methodology can be described as follows: for a mechanical model with $\mathrm{M}$ random variables, the response $\Gamma$ can be expressed by a PCE of order $p$ fixed by the user as follows:

$$
\Gamma_{P C E}(\xi)=\sum_{\beta=0}^{\infty} a_{\beta} \Psi_{\beta}(\xi) \cong \sum_{\beta=0}^{P-1} a_{\beta} \Psi_{\beta}(\xi)
$$

where $\xi$ is a vector of $M$ independent standard normal random variables representing the $M$ random variables, $P$ is the number of terms retained in the truncation scheme, $a_{\beta}$ are the unknown PCE coefficients to be computed and $\Psi_{\beta}$ are multivariate (or multidimensional) Hermite polynomials, which are orthogonal with respect to the joint probability density function of the standard normal random vector $\xi$. These multivariate Hermite polynomials can be obtained from the product of one-dimensional Hermite polynomials of the different random variables as follows:

$$
\Psi_{\beta}=\prod_{i=1}^{M} H_{\alpha_{i}}(\xi)
$$

where $H_{\alpha_{i}}($.$) is the \alpha_{\mathrm{i}}$-th one-dimensional Hermite polynomial and $\alpha_{i}$ is a sequence of $\mathrm{M}$ non-negative integers $\left\{\alpha_{1}, \ldots, \alpha_{M}\right\}$. The expressions of the one-dimensional Hermite polynomials can be found in [14] among others. In practice, one should truncate the PCE representation by retaining only the multivariate polynomials of degree less than or equal to the PCE order p. The classical truncation scheme suggests that the first order norm $\|.\|_{1}$ should be less than or equal to the order $p$ of the PCE as follows [18]:

$$
\|\alpha\|_{1}=\sum_{i=1}^{M} \alpha_{i} \leqslant p
$$

Using this method of truncation, the number $P$ of unknown PCE coefficients is given by

$$
P=\frac{(M+p) !}{M ! p !}
$$

As may be seen from Equation (13), the number P of the PCE coefficients, which is the number of terms retained in Equation (10), increases dramatically with the number $\mathrm{M}$ of random variables and 
the order $p$ of the PCE. This number becomes very high in the case of random fields where the number of random variables is significant. Hence, the SPCE methodology presented below is necessary in this case.

\subsection{Adaptive sparse polynomial chaos expansion-the hyperbolic (q-norm) truncation scheme}

Blatman and Sudret $[18,25,26]$ have shown that the number of significant terms in a PCE is relatively small because the multidimensional polynomials $\Psi_{\beta}$ corresponding to high-order interaction (i.e. those resulting from the multiplication of the $H_{\alpha_{i}}$ with increasing $\alpha_{i}$ values) are associated with very small values of coefficients $a_{\beta}$. These authors have also stated that the term resulting from the multiplication of the $H_{\alpha_{i}}$ with all $\alpha_{i}=0$ leads to a significant coefficient $a_{0}$ in the PCE. This coefficient represents the probabilistic mean value of the system response. Thus, a truncation strategy based on these observations was developed by Blatman and Sudret $[25,26]$ in which the multidimensional polynomials $\Psi_{\beta}$ corresponding to high-order interaction were penalized. This was performed by considering that the $q$-norm should be smaller than the PCE order as follows [26]:

$$
\left.\|\alpha\|_{q}=\sum_{i=1}^{M}\left(\alpha_{i}\right)^{q}\right)^{1 / q} \leqslant p
$$

where $q$ is a coefficient $(0<q<1)$. In this formula, $q$ can be chosen arbitrarily. Blatman and Sudret [26] have shown that sufficient accuracy is obtained for $q \geqslant 0.5$. Below this value, we may risk to reject some significant terms. The proposed SPCE strategy leads to a PCE that contains a small number of unknown coefficients, which can be calculated from a reduced number of calls of the deterministic model. This is of particular interest in the present case of random fields, which involves a significant number of random variables. An iterative procedure was suggested by Blatman and Sudret [18] for building up a SPCE. This procedure can be briefly described by the flowchart presented in Figure 1. It will be used in this paper to build up a SPCE of the system response. The method of computation of the coefficient of determination used in the flowchart can be described as follows: For an experimental design (ED) built up from a set of $\mathrm{K}$ realizations $\left\{\xi^{(1)}=\left(\xi_{1}, \ldots, \xi_{M}\right), \ldots, \xi^{(K)}=\left(\xi_{1}, \ldots, \xi_{M}\right)\right\}$ of the standard normal random vector $\xi$, the corresponding values of the system response denoted by $\Gamma=\left\{\Gamma\left(\xi^{(1)}\right), \ldots, \Gamma\left(\xi^{(K)}\right)\right\}$ are determined by deterministic calculations. These data are then used to compute the SPCE by regression. The accuracy of the response can be assessed using the coefficient of determination $R^{2}$ defined by the following:

$$
R^{2}=1-\Delta_{S P C E}
$$

where

$$
\begin{gathered}
\Delta_{S P C E}=\frac{\frac{1}{K} \sum_{i=1}^{K}\left(\Gamma\left(\xi^{(i)}\right)-\Gamma_{\text {SPCE }}\left(\xi^{(i)}\right)\right)^{2}}{\operatorname{Var}(\Gamma)} \\
\operatorname{Var}[\Gamma]=\frac{1}{K-1} \sum_{i=1}^{K}\left(\Gamma\left(\xi^{(i)}\right)-\bar{\Gamma}\right)^{2} \\
\bar{\Gamma}=\frac{1}{K} \sum_{i=1}^{K} \Gamma\left(\xi^{(i)}\right)
\end{gathered}
$$

The value $R^{2}=1$ indicates a perfect fit of the true model response $\Gamma$, whereas $R^{2}=0$ indicates a nonlinear relationship between the true model $\Gamma$ and the SPCE model $\Gamma_{S P C E}$. 


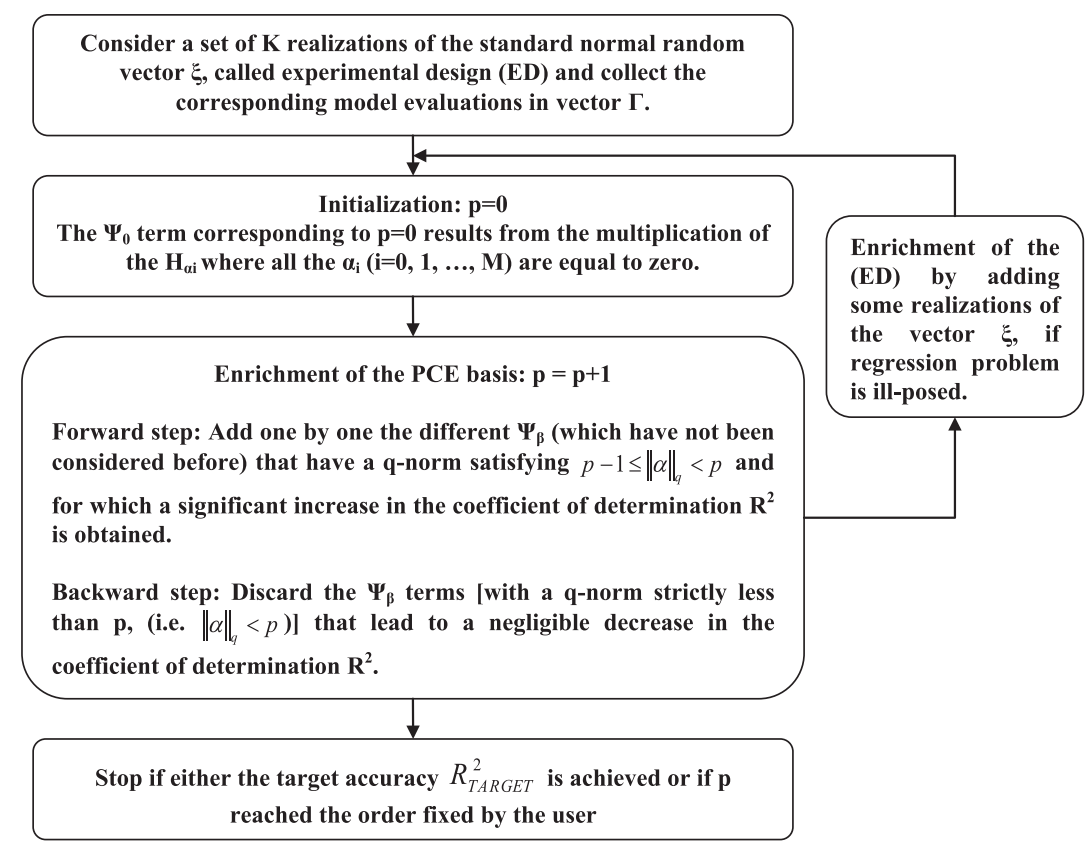

Figure 1. Flowchart used to build up a SPCE.

\subsection{Statistical analysis of the response}

Once the unknown coefficients of the SPCE are determined, the PDF of the system response and its corresponding statistical moments (i.e. mean $\mu$, standard deviation $\sigma$, skewness $\delta$, and kurtosis $\kappa$ ) can be estimated. This can be done by simulating a large number of realizations (e.g. six million simulations) of the independent standard normal random variables. Simulating a large number of realizations and their corresponding responses using the analytical formula dramatically reduces the computation time. It should be noticed here that the computation time required for the generation of a single realization and the computation of the corresponding system response is strongly related to the number of eigenmodes $N$ used in the discretization scheme. For very small autocorrelation distances (as will be seen later), the number of eigenmodes significantly increases, leading to a significant computation time (more than an hour for a single realization).

\subsection{Global sensitivity analysis}

The global sensitivity analysis (GSA) allows one to compute the weight of the different input random parameters in the variability of the system response. The GSA is generally based on the decomposition of the response variance as a sum of contributions of the different random variables or combinations of random variables. In the present paper where a random field is discretized into a finite number of random variables, a first-order sensitivity analysis involving the block vector of the standard Gaussian independent random variables $\xi=\left\{\xi_{c}=\left(\xi_{1}^{c}, \ldots, \xi_{N}^{c}\right), \xi_{\varphi}=\left(\xi_{1}^{\varphi}, \ldots, \xi_{N}^{\varphi}\right)\right\}$ is used. The sensitivity index of each random variable is first calculated. Then, the sensitivity index of each field (c or $\varphi$ ) is computed as the sum of all the indices of the variables of this field as follows:

$$
S_{i}=\sum_{j=1}^{N} \frac{\operatorname{Var}\left[E\left(\Gamma_{S P C E} \mid \xi_{j}^{i}\right)\right]}{\operatorname{Var}\left(\Gamma_{S P C E}\right)}
$$

where $i=c, \varphi$ and $\Gamma_{S P C E}$ is the system response given by the SPCE. Of particular interest in the PCE or SPCE methodology are the so-called Sobol indices [27] for estimating the sensitivity indices. It was shown in Sudret [24] that the Sobol sensitivity indices can be computed analytically from the 
coefficients of the PCE or SPCE of the model response. In this paper, the analytical formulas given by Sudret [24] were used to calculate the Sobol indices for the two random fields $c$ and $\varphi$.

\section{NUMERICAL RESULTS}

The aim of this section is to present the probabilistic numerical results. These results are provided in the case of a weightless soil, although the probabilistic analysis presented before remains valid if the soil weight was included in the analysis. The main reason for which the soil weight was neglected is the validation of the present probabilistic approach with the use a non-expensive deterministic model that has a reasonable computation time. Notice that introducing the soil weight in the deterministic model increases the computation time from 5 to 10 minutes per simulation. Although this difference may not seem to be significant for a single simulation, it becomes dramatically important during the probabilistic analyses where a large number of simulations is needed for each probabilistic analysis.

The deterministic model is based on numerical simulations using the finite difference code $\mathrm{FLAC}^{3 \mathrm{D}}$. The soil behavior is modeled using a conventional elastic-perfectly plastic model based on Mohr-Coulomb failure criterion. Because the friction angle $\varphi$ is bounded (i.e. $0 \leqslant \varphi \leqslant 45^{\circ}$ ), a beta distribution was selected for this parameter. This assumption was also adopted by several investigators (cf. [3, 28-31] among others). As for the cohesion $c$, non-negative values must be obtained for this parameter. Several authors (cf. [3, 28-31] among others) have suggested a lognormal distribution for the cohesion parameter. This assumption was also adopted in our paper. The mean values and coefficients of variation of the two random fields (referred to in this paper as reference values) are given as follows: $\mu_{c}=20 \mathrm{kPa}, \operatorname{Cov}_{c}=25 \% ; \mu_{\varphi}=30^{\circ}, \operatorname{Cov}_{\varphi}=10 \%$. The dilation angle $\psi$ (associated to the rate of plastic dilation) is considered to be related to the friction angle $\varphi$ by $\psi=\frac{2}{3} \varphi$. This means that the dilation angle was implicitly assumed as a random field that is perfectly correlated to the friction angle field. To incorporate the dependence between the shear strength parameters, the cross-correlation coefficient $r(c, \varphi)$ is needed. Yucemen et al. [32] reported values that are in a range of $-0.49 \leqslant r \leqslant-0.24$, whereas Lumb [33] suggested values of $-0.7 \leqslant r \leqslant-0.37$. In this study, a value of -0.5 was taken as the reference value, and the range of $-0.5 \leqslant r \leqslant 0$ was considered in the parametric study. The reference cross-correlation matrix between the two random fields $(c, \varphi)$ is thus given by the following:

$$
C^{N G}=\left(\begin{array}{cc}
1 & -0.5 \\
-0.5 & 1
\end{array}\right)
$$

Finally, notice that Young modulus $\mathrm{E}$ and Poisson ratio $\mathrm{v}$ were assumed to be deterministic because the ultimate bearing capacity is not sensitive to these variables. Their corresponding values are $E=60 M P a$ and $v=0.3$, respectively. Finally, concerning the footing, a weightless strip foundation of $2 \mathrm{~m}$ width and $0.5 \mathrm{~m}$ height is used. It is assumed to follow an elastic linear model $(E=25 G P a, v=0.4)$. The connection between the footing and the soil mass is modeled by interface elements having the same mean values of the soil shear strength parameters to simulate a perfectly rough soil-footing interface. These parameters have been considered as deterministic in this study. Concerning the elastic properties of the interface, they also have been considered as deterministic, and their values are as follows: $K_{s}=1 G P a, K_{n}=1 G P a$ where $K_{\mathrm{s}}$ and $K_{\mathrm{n}}$ are the shear and normal stiffness, respectively.

As for the autocorrelation distances $a_{x}$ and $a_{y}$ of the two random fields $c$ and $\varphi$, both cases of isotropic (i.e. $a_{x}=a_{y}$ ) and anisotropic (i.e. $a_{x} \# a_{y}$ ) autocorrelation distances will be treated, although the soil is rarely isotropic in reality. For the isotropic case, a range of $1.5-100 \mathrm{~m}$ was considered. For the anisotropic case, El-Ramly et al. [34] have shown that $a_{x}$ is within a range of $10-40 \mathrm{~m}$, whereas $a_{y}$ ranges from 1 to $3 \mathrm{~m}$. These values are in accordance with those given by Phoon and Kulhawy [35]. In our study, the reference values adopted for $a_{x}$ and $a_{y}$ were $a_{x}=10 \mathrm{~m}$ and $a_{y}=1 \mathrm{~m}$, whereas the wide ranges of 2-50 m and $0.5-8 \mathrm{~m}$ were considered respectively for $a_{x}$ and $a_{y}$ when performing the parametric study to explore the possible existence of a minimum value of the probabilistic mean. 
As shown in Figure 2, the adopted soil domain considered in the analysis is $15 \mathrm{~m}$ wide by $6 \mathrm{~m}$ deep. It should be noted that the size of a given element in the deterministic mesh depends on the autocorrelation distances of the soil properties. Der Kiureghian and Ke [36] have suggested that the length of the largest element of the deterministic mesh in a given direction (horizontal or vertical) should not exceed 0.5 times the autocorrelation distance in that direction. To respect this criterion for the different autocorrelation distances, two different deterministic meshes were considered in FLAC ${ }^{3 \mathrm{D}}$. The first one is devoted to the case of moderate to great values of the autocorrelation distances (i.e. when $a_{x} \geqslant 10 \mathrm{~m}$ and $a_{y} \geqslant 1 \mathrm{~m}$ ) [see Figure 2(a)] and the second one for the small values of the autocorrelation distances (i.e. when $1.5 m \leqslant a_{x}<10 m$ or $0.5 m \leqslant a_{y}<1 m$ ) [see Figure 2(b)]. For the boundary conditions, the horizontal movement on the vertical boundaries of the grid is restrained, whereas the base of the grid is not allowed to move in both the horizontal and the vertical directions.

The following sections are organized as follows: First, a brief description of a step-by-step procedure used to obtain the probabilistic results is presented. It is followed by the presentation of some realizations of the random fields and the PDFs of the system responses. Finally, the effect of the different probabilistic governing parameters on the PDF of the ultimate bearing capacity is presented and discussed.

\subsection{Step-by-step procedure used for the computation of the probabilistic results}

A Matlab 7.0 code was implemented to obtain the probabilistic results. The different steps of this code in the general case of two anisotropic cross-correlated non-Gaussian random fields are as follows:

(a) Introduce the input statistical parameters given in the preceding section.

(b) Discretize the two random fields $c$ and $\varphi$ using EOLE method and its extensions by Vořechovsky [19] as follows:

- Define the stochastic grid: Li and Der Kiureghian [20] have shown that the variance of the error (Equation 9) is large at the boundaries of the stochastic domain. This problem can be solved using a stochastic domain $\Omega_{\mathrm{RF}}$ that extends beyond the boundaries of the physical domain $\Omega$. In this paper, a uniform stochastic grid of dimensions $\Omega_{\mathrm{RF}}=[16 \mathrm{~m}, 7 \mathrm{~m}]$ was used, whereas the size of the physical domain was $\Omega=[15 \mathrm{~m}, 6 \mathrm{~m}]$. On the other hand, $\mathrm{Li}$ and Der Kiureghian [20] have shown that the number of grid-points in the stochastic grid strongly depends on the autocorrelation distances. These authors have shown that a ratio of about $\frac{l_{R F}}{a}=\frac{1}{5}$ provides a sufficient accuracy in terms of the variance of the error where $l_{R F}$ is the typical element size in the stochastic grid and $a$ is the autocorrelation distance. In this paper, the number of grid points in the stochastic grid was chosen as follows: six grid points were considered within each autocorrelation distance (horizontal or vertical) with a minimum of six grid points in that direction when the autocorrelation distance is larger than the size of the stochastic domain. Thus, a fine stochastic mesh was used for a highly heterogeneous soil, and a coarse stochastic mesh was used for a slightly heterogeneous soil.

- Calculate the common autocorrelation matrix $\Sigma_{\chi ; \alpha}^{N G}$ using Equation (2) (notice that the dimensions of this matrix depend on the values of the autocorrelation distances $a_{x}$ and $a_{y}$ ). Then, compute the corresponding autocorrelation matrices $\Sigma_{\chi ; \chi}^{c}$ and $\Sigma_{\chi ; \chi}^{\varphi}$ in the Gaussian space using Nataf model (Equation 3). It should be mentioned here that both matrices $\Sigma_{\chi ; \chi}^{c}$ and $\Sigma_{\chi ; \chi}^{\varphi}$ were quasi-similar

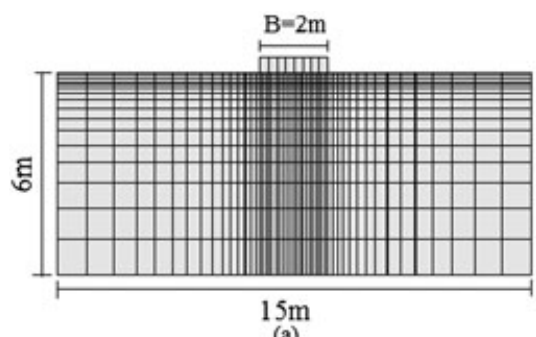

(a)

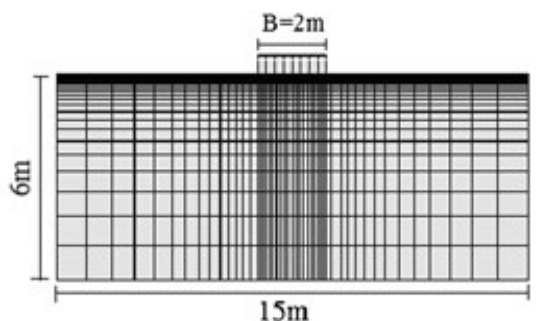

(b)

Figure 2. Mesh used for the computation of the ultimate bearing capacity: (a) for moderate to great values of the autocorrelation distances $\left(a_{\mathrm{x}} \geqq 10 \mathrm{~m}\right.$ and $\left.a_{\mathrm{y}} \geqq 1 \mathrm{~m}\right)$, (b) for small values of the autocorrelation distances $\left(a_{\mathrm{x}}<10 \mathrm{~m}\right.$ or $\left.a_{\mathrm{y}}<1 \mathrm{~m}\right)$. 
to $\Sigma_{\gamma: \mathcal{r}}^{N G}$, and thus, the number of eigenmodes (number of random variables), which is necessary to discretize each one of the two random fields, was similar. Finally, compute for each random field ( $c$ and $\varphi$ ) its $N$ largest eigenmodes $\lambda_{j}^{i}$ and $\varphi_{j}^{i}$ (where $i=c, \varphi$ and $j=1, \ldots, N$ ), for which the variance of the error is smaller than a threshold of say $\varepsilon \approx 10 \%$. As may be seen from Figure 3 , for smaller values of the autocorrelation distance $\left(a_{x}, a_{y}\right.$ or $\left.a_{x}=a_{y}\right)$, the number $N$ of eigenmodes increases. The total number of random variables retained for different cases (where two random fields were considered) is presented in Table I. This number is equal to the number of eigenmodes $N$ of a single random field multiplied by 2 because two random fields were considered in the analysis. It should be noticed that the cases where a significant number of random variables $(>88)$ are needed correspond to very small autocorrelation distances (i.e. $a_{y}<1 \mathrm{~m}$ and $a_{x}<2 \mathrm{~m}$ ). These autocorrelation distances are not of practical interest [see El-Ramly et al. [34]] and can thus be neglected in this study.

- Calculate the Gaussian cross-correlated matrix $C$ by applying the Nataf model on the original non-Gaussian cross-correlation matrix $C^{N G}$ given by Equation (20). This was performed using Equation (4).

- Discretize the two anisotropic cross-correlated Gaussian fields c and $\varphi$ using Equations (5) and (6) where $\kappa^{D}$ was computed using Equation (7), the transformation to the non-Gaussian space being done by applying Equation (8).

(c) Use the adaptive SPCE methodology to determine the meta-model as follows: First, it should be noted that for each realization, the values of the two random fields $(c$ and $\varphi)$ were determined at the centroid of each element of the deterministic mesh. Once the different elements of the mesh are filed with values of $c$ and $\varphi$, the ultimate bearing capacity $q_{\mathrm{ult}}$ for this specific realization can

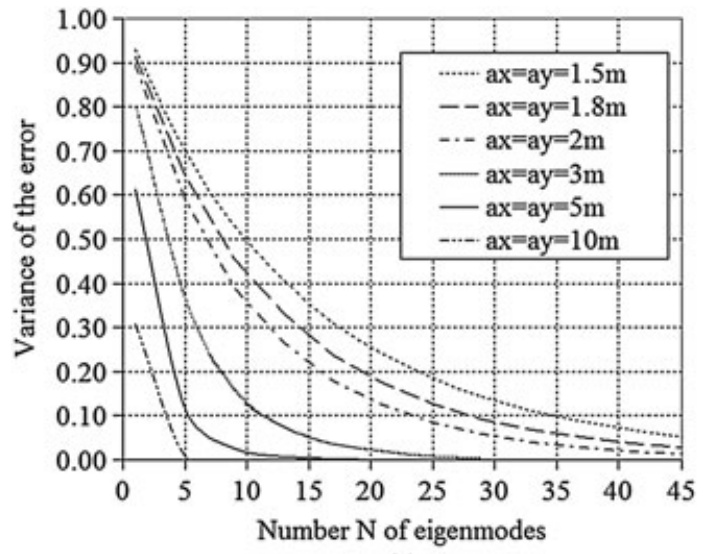

(a)

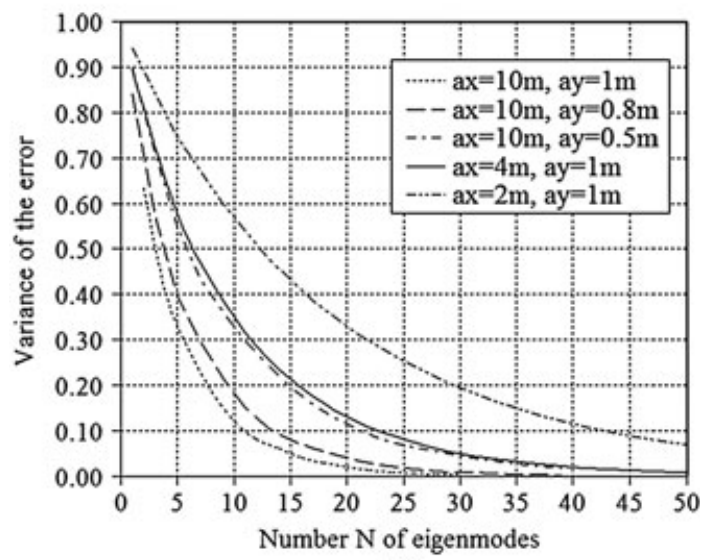

(b)

Figure 3. Number $\mathrm{N}$ of eigenmodes needed in the EOLE method: (a) isotropic case, (b) anisotropic case. 
Table I. Number of random variables used to discretize the two random fields $c$ and $\varphi$ for both cases of isotropic and anisotropic autocorrelation distances.

\begin{tabular}{|c|c|c|}
\hline & & $\begin{array}{l}\text { Total number of random variables used to discretize the } \\
\text { two random fields }(c, \varphi)\end{array}$ \\
\hline \multirow[t]{6}{*}{ Isotropic case } & $a_{x}=a_{y}=1.5 m$ & 70 \\
\hline & $a_{x}=a_{y}=1.8 m$ & 60 \\
\hline & $a_{x}=a_{y}=2 m$ & 50 \\
\hline & $a_{x}=a_{y}=3 m$ & 24 \\
\hline & $a_{x}=a_{y}=5 m$ & 20 \\
\hline & $a_{x}=a_{y}=10 m$ & 10 \\
\hline \multirow[t]{5}{*}{ Anisotropic case } & $a_{x}=10 m, a_{y}=1 m$ & 24 \\
\hline & $a_{x}=10 m, a_{y}=0.8 m$ & 30 \\
\hline & $a_{x}=10 m, a_{y}=0.5 m$ & 44 \\
\hline & $a_{x}=4 m, a_{y}=1 m$ & 48 \\
\hline & $a_{x}=2 m, a_{y}=1 m$ & 88 \\
\hline
\end{tabular}

be determined. The experimental design (ED) was obtained by first simulating an arbitrary number of realizations $\mathrm{K}=200$ of the two random fields $(c$ and $\varphi$ ) using MCS technique. $\mathrm{K}$ is an initial (arbitrary) value because the adaptive algorithm will automatically add other simulations (an arbitrary number of 100 realizations) each time the regression problem is ill posed (i.e. when the rank of the matrix used in the regression approach is smaller than the number of unknown coefficients). The algorithm stops if either the target accuracy $R_{T A R G E T}^{2}$ is achieved or if $p$ reached the order fixed by the user. In this paper, a target accuracy $R_{\text {TARGET }}^{2}=$ 0.999 , a coefficient $q=0.7$, and a maximal order $p=5$ were used. Notice that for the reference case $\left[a_{x}=10 \mathrm{~m}, a_{y}=1 \mathrm{~m}, \mathrm{r}(\mathrm{c}, \varphi)=-0.5\right]$, the algorithm have stopped when the target accuracy was reached. The corresponding order of the SPCE was equal to 3. In this case, where 24 random variables were needed (see Table I), the PCE in its 'full' truncation schemes leads to $P=2925$ unknown coefficients. This means that a minimum of 2925 collocation points (i.e. a minimum of 2925 calls of the deterministic model) were needed to accurately represent the ultimate bearing capacity by a meta-model. Using the SPCE methodology, only $P=186$ unknown coefficients were retained, and only 800 Monte Carlo simulations were found to be largely sufficient to construct the meta-model. Consequently, a reduction of minimum $50 \%$ in the number of calls of the deterministic model can be obtained using the SPCE. This greatly facilitates the solution of the problem of random fields.

(d) Use the meta-model to perform the post-treatment. This consists in determining the following: (i) the PDF of the ultimate bearing capacity and the corresponding statistical moments (mean, variance, skewness and kurtosis) and (ii) the Sobol indices for each random field ( $c$ and $\varphi$ ).

Finally, it should be mentioned that a link between Matlab and FLAC $^{3 \mathrm{D}}$ was performed to automatically exchange the data in both directions and thus to decrease the computation time.

\subsection{Random fields' realizations and probability density functions of the system responses}

Figure 4 presents six realizations for three different configurations. As may be seen from this figure, the anisotropy and the negative cross-correlation are well reflected by the obtained random realizations.

Figure 5 presents the PDFs of the footing ultimate bearing capacity and the footing rotation for the reference case where $a_{x}=10 \mathrm{~m}, a_{y}=1 \mathrm{~m}$, and $r(c, \varphi)=-0.5$. Figure 6 presents the velocity field for one single simulation (i.e. a single realization of the two random fields $c$ and $\varphi$ ). As may be seen from this figure, the spatial variability of soil properties can produce a non-symmetrical mechanism, although the footing is subjected to a symmetrical vertical load. Although the footing rotation of a single realization is not null as may be seen from Figure 6, the mean value of the rotation for the whole number of realizations is null [see Figure 5(b)], and the standard deviation of this rotation was found equal to $1.6 \times 10^{-4}$ radians. 

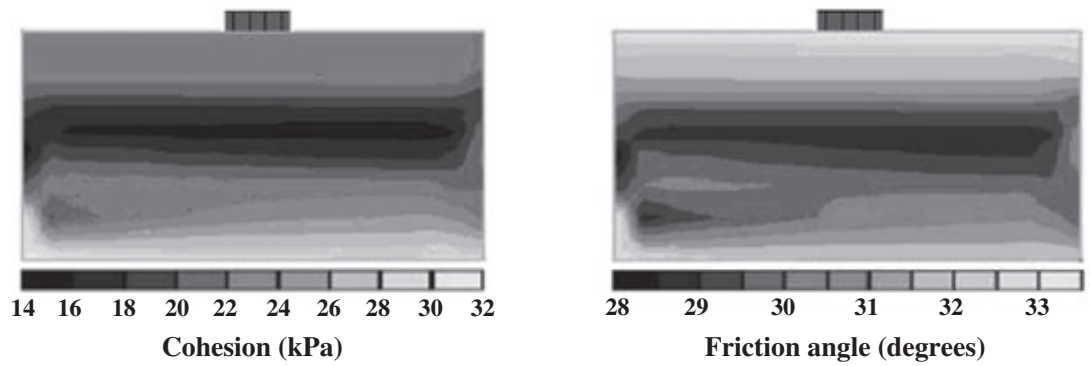

(a)
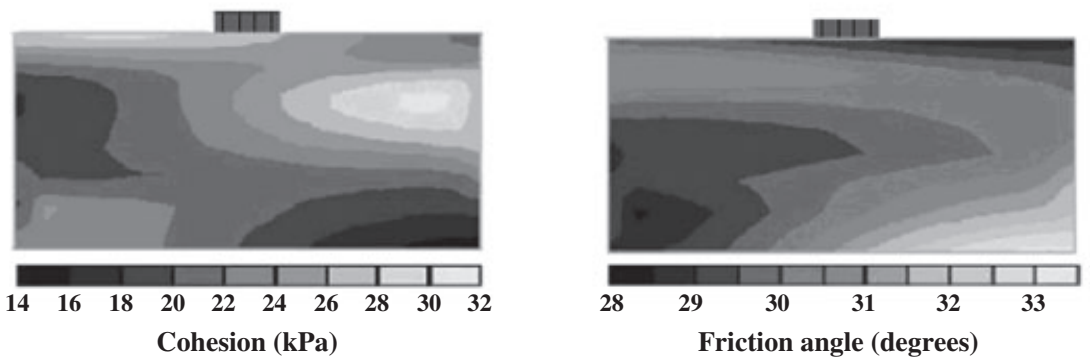

(b)
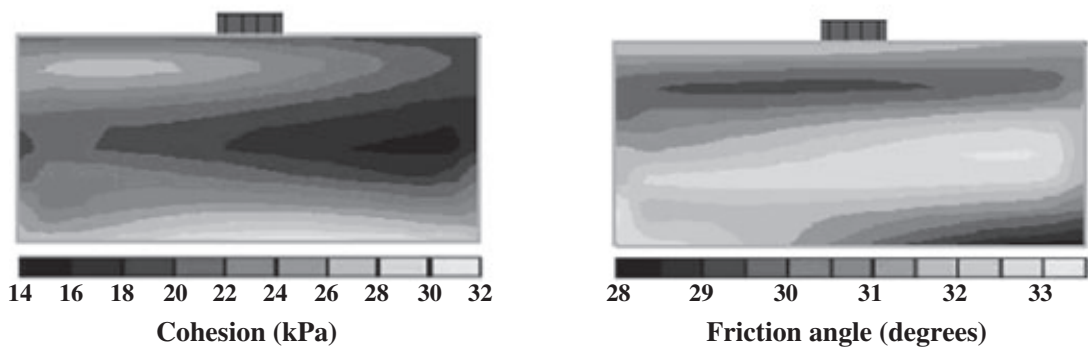

(c)

Figure 4. Typical realizations of the random fields: (a) $\left[a_{\mathrm{x}}=100 \mathrm{~m}, a_{\mathrm{y}}=1 \mathrm{~m}, r(c, \varphi)=-0.5\right]$; (b) $\left[a_{\mathrm{x}}=10 \mathrm{~m}\right.$, $\left.a_{\mathrm{y}}=1 \mathrm{~m}, r(c, \varphi)=-0.5\right]$; (c) $\left[a_{\mathrm{x}}=10 \mathrm{~m}, a_{\mathrm{y}}=1 \mathrm{~m}, r(c, \varphi)=-0.9\right]$.

\subsection{Probabilistic parametric study}

The aim of this section is to study the effect of the different probabilistic governing parameters (autocorrelation distances, coefficients of variation) of the two random fields and the correlation between both fields on the PDF of the ultimate bearing capacity of the foundation.

3.3.1. Effect of the autocorrelation distance: the isotropic case. Figure 7 provides the PDFs of the ultimate bearing capacity for different values of the isotropic autocorrelation distance $a_{x}=a_{y}(1.5$, $1.8,2,3,5,10 \mathrm{~m})$ when $r(c, \varphi)=-0.5$. Table II presents the four statistical moments for the cases presented in Figure 7 together with those corresponding to great values of the autocorrelation distance including the case of random variables. Figure 7 shows that the PDF is less spread out when the autocorrelation distance decreases. On the other hand, for large values of the autocorrelation distance (see Table II), the standard deviation tends to the value obtained in the case of a homogeneous soil (case of random variables). The variability of the ultimate bearing capacity decreases with the increase in the soil heterogeneity because the zone involved by the possible failure mechanism will have average values of the shear strength parameters close to the mean values of the two fields because of the large number of high and small values of the shear strength parameters. This leads to close values of the ultimate bearing capacity and thus to a smaller variability in this bearing capacity.

Figure 8 and Table II show that the probabilistic mean value of the ultimate bearing capacity presents a minimum when the autocorrelation distance $a_{x}=a_{y}$ is nearly equal to the footing breadth B (i.e. in our case, when $a_{x}=a_{y}=2 \mathrm{~m}$ ). Notice that the minimal probabilistic mean was also 


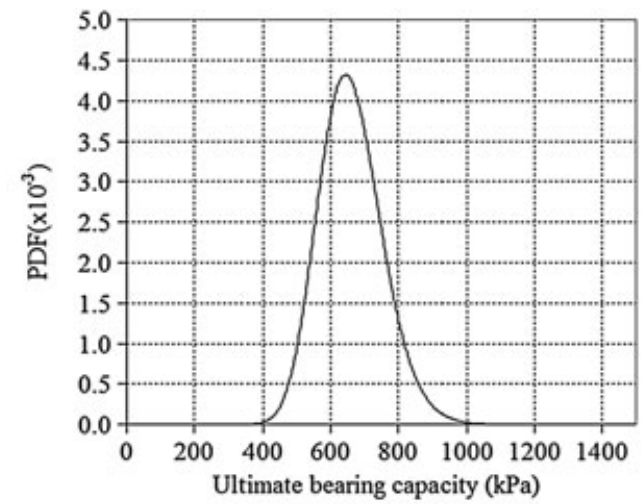

(a)

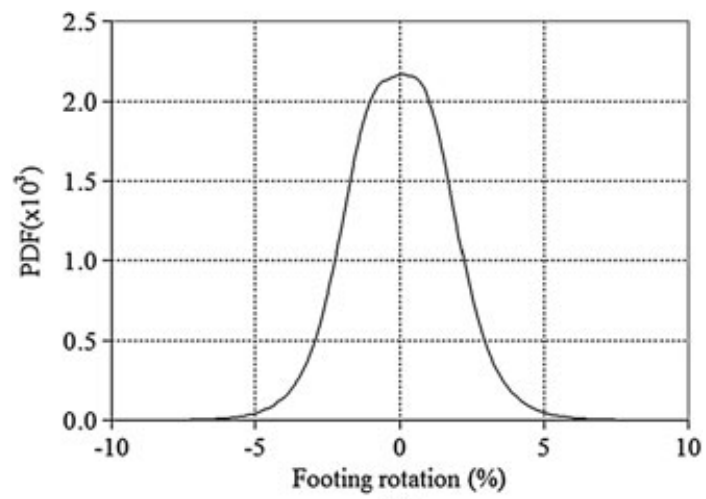

(b)

Figure 5. Bearing capacity and footing rotation of the reference case where $a_{\mathrm{x}}=10 \mathrm{~m}, a_{\mathrm{y}}=1 \mathrm{~m}$, and $r(c, \varphi)=-0.5$ : (a) PDF of the ultimate bearing capacity; and (b) PDF of the footing rotation.

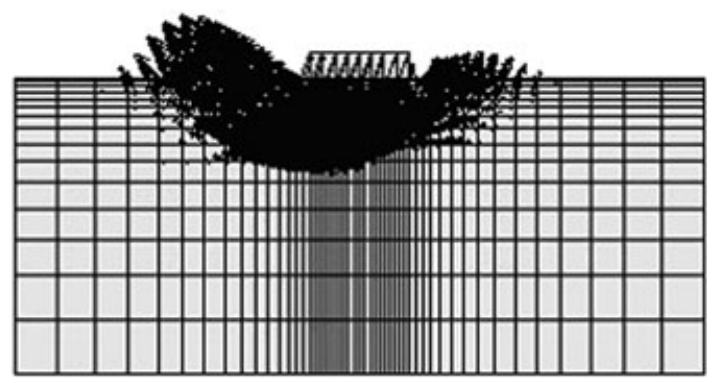

Figure 6. Velocity field for a typical realization of the two random fields for the reference case where $a_{\mathrm{x}}=10 \mathrm{~m}, a_{\mathrm{y}}=1 \mathrm{~m}, r(c, \varphi)=-0.5$.

observed by Fenton and Griffiths [3] and Soubra et al. [6] when isotropic random fields were studied. For very large autocorrelation distances $\left(a_{x}=a_{y}=100 \mathrm{~m}\right)$, the probabilistic mean tends to the one of the homogenous soil (case of random variables) as may be seen from Table II. On the other hand, for very small autocorrelation distances the probabilistic mean becomes greater than the minimal value because the weakest path becomes increasingly tortuous and its length is also longer. As a result, the failure mechanisms will start to look for shorter path cutting through higher values of the shear strength parameters.

Table II also shows the impact of the autocorrelation distance $a_{x}=a_{y}$ on both the skewness and the kurtosis of the PDF. For small values of $a_{x}=a_{y}$, the skewness and kurtosis of the response are small, which means that the PDF of the response is not far from a Gaussian one in these cases. Notice however that these moments increase when $a_{x}=a_{y}$ increases which means that for great values of 


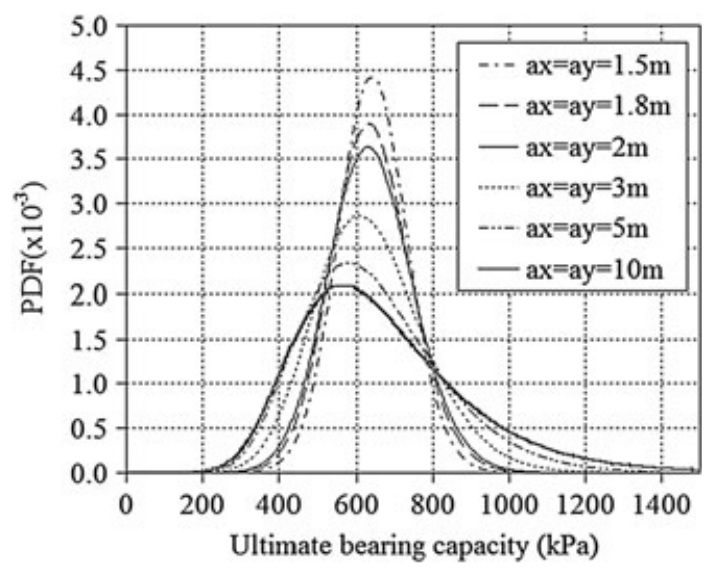

Figure 7. Influence of the isotropic autocorrelation distance $a_{\mathrm{x}}=a_{\mathrm{y}}$ on the PDF of the ultimate bearing capacity in the case where $r(c, \varphi)=-0.5$.

Table II. Effect of the isotropic autocorrelation distance $a_{\mathrm{x}}=a_{\mathrm{y}}$ on the statistical moments of the ultimate bearing capacity.

\begin{tabular}{lccccc}
\hline & $\mu_{q_{u t}}(\mathrm{kPa})$ & $\sigma_{q_{u t}}(\mathrm{kPa})$ & $\mathrm{COV} \%$ & $\delta_{u}(-)$ & $\kappa_{u}(-)$ \\
\hline$a_{x}=a_{y}=1.5 m$ & 642.6 & 88.85 & 13.83 & 0.063 & 0.083 \\
$a_{x}=a_{y}=1.8 \mathrm{~m}$ & 639.83 & 101.36 & 15.84 & 0.186 & 0.132 \\
$a_{x}=a_{y}=2 \mathrm{~m}$ & 638.74 & 108.86 & 17.04 & 0.197 & 0.128 \\
$a_{x}=a_{y}=3 \mathrm{~m}$ & 639.58 & 138.80 & 21.70 & 0.398 & 0.302 \\
$a_{x}=a_{y}=5 \mathrm{~m}$ & 646.45 & 175.85 & 27.20 & 0.671 & 0.656 \\
$a_{x}=a_{y}=10 \mathrm{~m}$ & 670.01 & 217.70 & 32.50 & 0.924 & 1.476 \\
$a_{x}=a_{y}=50 \mathrm{~m}$ & 676.54 & 227.39 & 33.61 & 1.072 & 1.926 \\
$a_{x}=a_{y}=100 \mathrm{~m}$ & 680.75 & 229.91 & 33.77 & 1.083 & 2.026 \\
Random variables & 682.67 & 232.8 & 32.14 & 1.093 & 2.475 \\
\hline
\end{tabular}

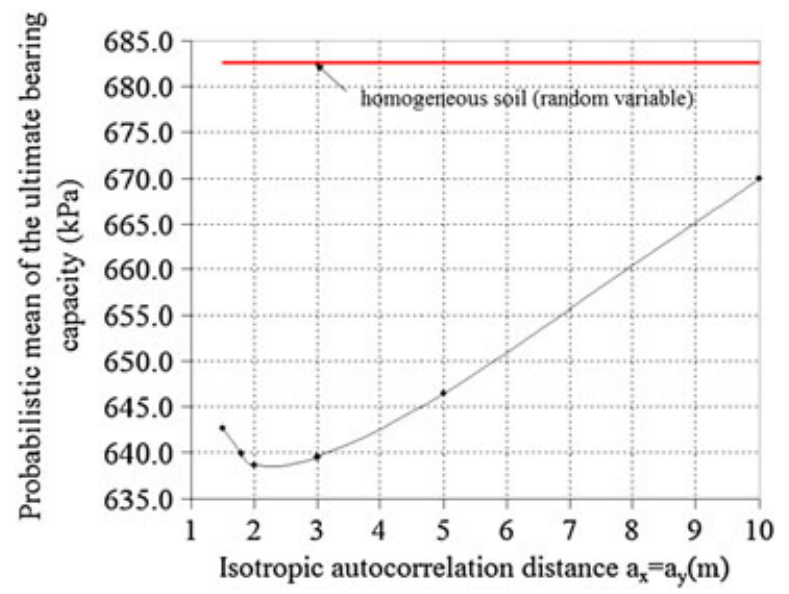

Figure 8. Influence of the isotropic autocorrelation distance $a_{\mathrm{x}}=a_{\mathrm{y}}$ on the probabilistic mean value of the ultimate bearing capacity in the case where $r(c, \varphi)=-0.5$.

$a_{x}=a_{y}$, the shape of the PDF of the output becomes far from a Gaussian one (the point of maximum density of probability, i.e. the mode moves to smaller values).

Finally, Table III shows the effect of the autocorrelation distance $a_{x}=a_{y}$ on the Sobol indices $S(\mathrm{c})$ and $S(\varphi)$ of the two random fields $c$ and $\varphi$. This table shows that both indices are quasi-constant 
Table III. Effect of the isotropic autocorrelation distance $a_{\mathrm{x}}=a_{\mathrm{y}}$ on the Sobol indices of the two random fields $c$ and $\varphi$.

\begin{tabular}{llr}
\hline$a_{x}=a_{y}(\mathrm{~m})$ & $S(c)$ & $S(\varphi)$ \\
\hline$a_{x}=a_{y}=1.5 \mathrm{~m}$ & 0.702 & 0.298 \\
$a_{x}=a_{y}=1.8 \mathrm{~m}$ & 0.710 & 0.290 \\
$a_{x}=a_{y}=2 \mathrm{~m}$ & 0.721 & 0.279 \\
$a_{x}=a_{y}=3 \mathrm{~m}$ & 0.728 & 0.272 \\
$a_{x}=a_{y}=5 \mathrm{~m}$ & 0.714 & 0.286 \\
$a_{x}=a_{y}=10 \mathrm{~m}$ & 0.726 & 0.274 \\
$a_{x}=a_{y}=50 \mathrm{~m}$ & 0.705 & 0.295 \\
$a_{x}=a_{y}=100 \mathrm{~m}$ & 0.706 & 0.294 \\
Random variables & 0.692 & 0.308 \\
\hline
\end{tabular}

regardless of the autocorrelation distance values. The increase in $a_{x}=a_{y}$ has no significant impact on the Sobol indices because we increase $a_{x}=a_{y}$ in both fields by the same amount. Table III also shows that the variability of the ultimate bearing capacity is mainly due to the cohesion random field, which has a Sobol index of about $71 \%$. This result is logical in our case where a weightless soil was considered, the $\mathrm{N} \gamma$ term, which is very sensitive to $\varphi$ being absent in this paper.

3.3.2. Effect of the autocorrelation distances: the anisotropic case. Figure 9 presents the PDFs of the ultimate bearing capacity for different values of $a_{y}\left(a_{y}=0.5,0.8,1,2,5\right.$, and $\left.8 \mathrm{~m}\right)$ when $a_{x}=10 \mathrm{~m}$ and $r(c, \varphi)=-0.5$, and Table IV presents the corresponding four statistical moments. Figure 9 and Table IV show that the variability of the ultimate bearing capacity decreases when the vertical autocorrelation distance $a_{y}$ decreases. The same explanation given in the isotropic case can be done here. Similarly, Figure 10 presents the PDFs of the ultimate bearing capacity for different values of $a_{x}\left(a_{x}=2,4,10,20,30\right.$ and $\left.50 \mathrm{~m}\right)$ when $a_{y}=1 \mathrm{~m}$ and $r(c, \varphi)=-0.5$, and Table $\mathrm{V}$ presents

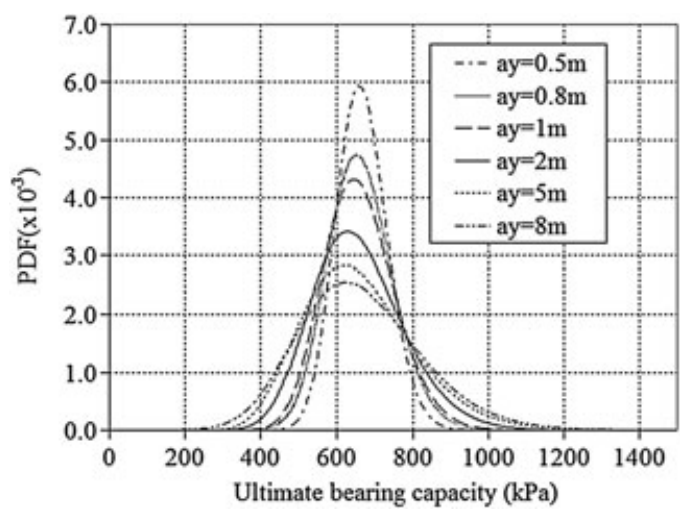

Figure 9. Influence of the vertical autocorrelation distance $\mathrm{a}_{\mathrm{y}}$ on the PDF of the ultimate bearing capacity in the case where $r(c, \varphi)=-0.5$ and $a_{\mathrm{x}}=10 \mathrm{~m}$.

Table IV. Effect of the vertical autocorrelation distance $a_{\mathrm{y}}$ on the statistical moments of the ultimate bearing capacity.

\begin{tabular}{lccccc}
\hline$a_{y}(\mathrm{~m})$ & $\mu_{q_{u t t}}(\mathrm{kPa})$ & $\sigma_{q_{u t}}(\mathrm{kPa})$ & $\mathrm{COV} \%$ & $\delta_{u}(-)$ & $\kappa_{u}(-)$ \\
\hline 0.5 & 665.50 & 67.65 & 10.16 & 0.198 & 0.089 \\
0.8 & 662.12 & 83.73 & 12.65 & 0.271 & 0.136 \\
1 & 658.20 & 93.57 & 14.22 & 0.287 & 0.163 \\
2 & 660.60 & 120.71 & 18.30 & 0.423 & 0.263 \\
5 & 661.00 & 147.33 & 22.30 & 0.546 & 0.446 \\
8 & 662.20 & 148.66 & 26.78 & 0.615 & 0.545 \\
\hline
\end{tabular}




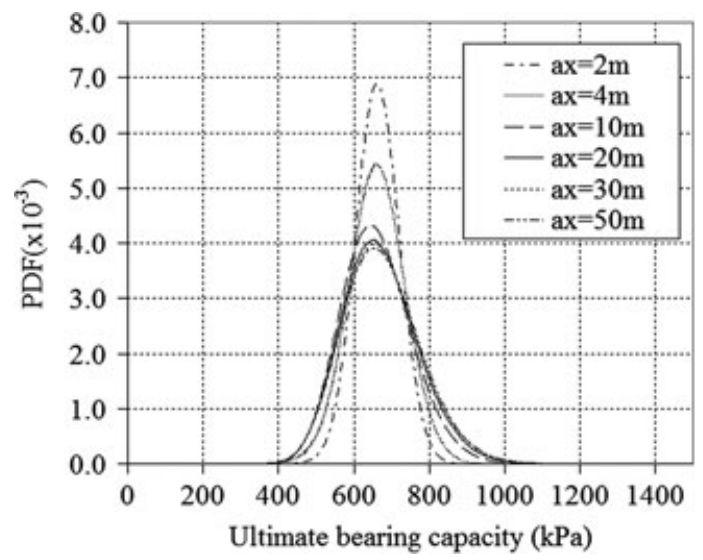

Figure 10. Influence of the horizontal autocorrelation distance $a_{\mathrm{x}}$ on the PDF of the ultimate bearing capacity in the case where $r(c, \varphi)=-0.5$ and $a_{\mathrm{y}}=1 \mathrm{~m}$.

Table V. Effect of the horizontal autocorrelation distance $a_{\mathrm{x}}$ on the statistical moments of the ultimate bearing capacity.

\begin{tabular}{lccccc}
\hline$a_{x}(m)$ & $\mu_{q_{u t}}(\mathrm{kPa})$ & $\sigma_{q_{u t}}(\mathrm{kPa})$ & $\mathrm{COV} \%$ & $\delta_{u}(-)$ & $\kappa_{u}(-)$ \\
\hline 2 & 662.73 & 55.68 & 8.40 & 0.023 & 0.054 \\
4 & 660.22 & 72.14 & 10.92 & 0.025 & 0.112 \\
10 & 658.18 & 93.56 & 14.21 & 0.287 & 0.163 \\
20 & 669.81 & 100.21 & 14.96 & 0.379 & 0.231 \\
30 & 673.30 & 102.65 & 15.24 & 0.391 & 0.265 \\
50 & 675.24 & 103.70 & 15.36 & 0.395 & 0.238 \\
\hline
\end{tabular}

the corresponding four statistical moments. The same observations concerning the variability of the ultimate bearing capacity made before (when varying $a_{y}$ ) remain valid in the present case. Notice, however, that beyond a value of $a_{x}=20 \mathrm{~m}$, the horizontal autocorrelation distance has a small effect on the variability of the ultimate bearing capacity.

Figures 11 and 12 and Tables IV and V show that the probabilistic mean value of the ultimate bearing capacity presents a minimum at a certain value of the ratio $a_{x} / a_{y}$ (in our paper, this value is equal to 10 for the prescribed values of the soil and footing characteristics). The presence of a minimum value can be explained as follows:

For a prescribed value of the horizontal autocorrelation distance $a_{x}$, the very small value of the vertical autocorrelation distance $a_{y}$ (i.e. corresponding to $a_{x} / a_{y}>>1$ ) creates a horizontal multilayer composed of very thin sublayers for which each sublayer may have a large or a small value of the shear strength parameters [see Figure 13(a)]. On the other hand, the very large value of the vertical autocorrelation distance $a_{y}$ (i.e. corresponding to $a_{x} / a_{y}<<1$ ) leads to a vertical multilayer (case of a one-dimensional horizontal random field) composed of a finite number of sublayers for which each sublayer may have a large or a small value of the shear strength parameters [see Figure 13(b)]. For both cases of very small and very large values of $a_{y}$, the variety of sublayers with large and small values of the shear strength parameters leads to a greater value of the ultimate bearing capacity. This large value occurs because the sublayers having large values of the shear strength parameters play the role of an obstacle. In fact, the failure mechanisms will cut sublayers having large values of the soil shear strength parameters. Finally, for medium autocorrelation distances [see Figure 13(e)], the soil contains a number of stiff zones adjacent to a number of soft zones whose areas are less extended in both the vertical and the horizontal directions compared to the two previous cases. This allows the development of the failure mechanism through the soft soil zones and thus, this leads to smaller values of the ultimate bearing capacity. 


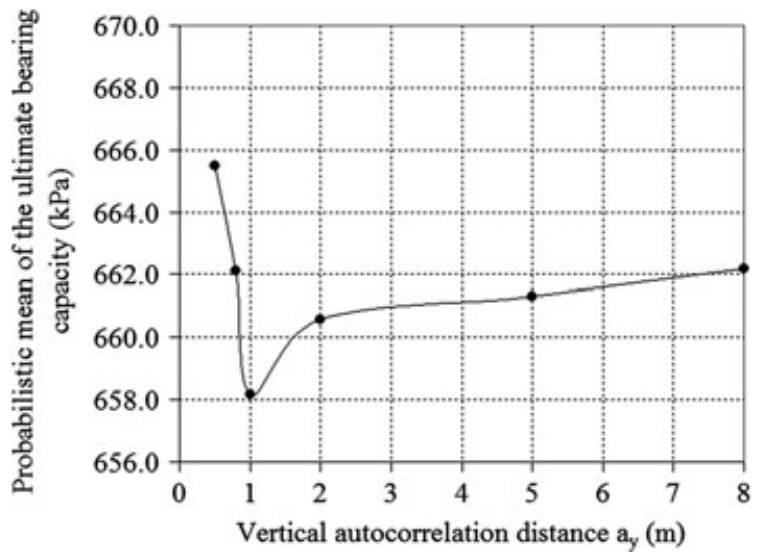

Figure 11. Influence of the vertical autocorrelation distance $a_{\mathrm{y}}$ on the probabilistic mean value of the ultimate bearing capacity in the case where $r(c, \varphi)=-0.5$ and $a_{\mathrm{x}}=10 \mathrm{~m}$.

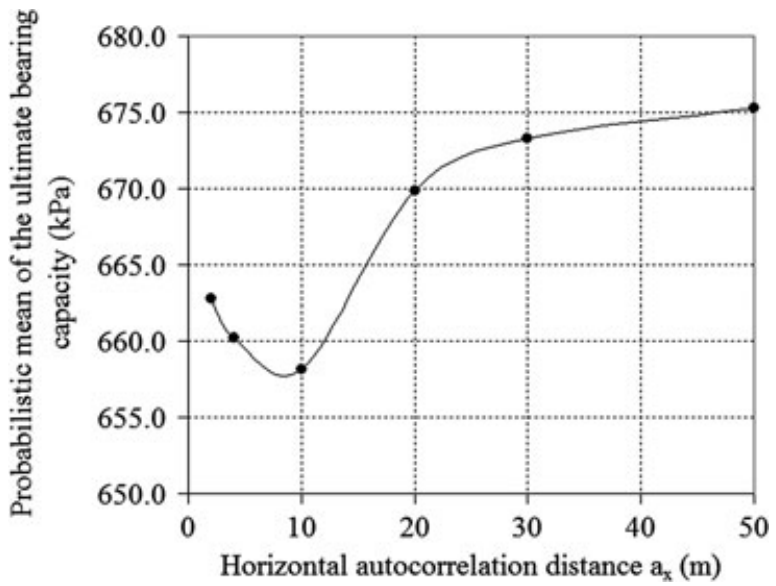

Figure 12. Influence of the horizontal autocorrelation distance $a_{\mathrm{x}}$ on the probabilistic mean value of the ultimate bearing capacity in the case where $r(c, \varphi)=-0.5$ and $a_{\mathrm{y}}=1 \mathrm{~m}$.

Similar to Figure 11, Figure 12 shows that for a prescribed value of the vertical autocorrelation distance $a_{y}$, the very small value of the horizontal autocorrelation distance $a_{x}$ leads to a vertical multilayer composed of a large number of thin sublayers for which each sublayer may have a large or a small value of the shear strength parameters [see Figure 13(c)]. Also, a horizontal multilayer is obtained in the case of a very large value of $a_{x}$ [see Figure 13(d)]. Finally, a soil composed of several soft and stiff zones of finite dimensions is obtained for intermediate values of the autocorrelation distances [see Figure 13(e)]. For all the three cases corresponding to small, intermediate and high values of the horizontal autocorrelation distance, the explanation given for Figure 11 remains valid herein.

As a conclusion, one may observe that the increase in the autocorrelation distance in Figure 11 leads to a soil configuration that varies from a horizontal to a vertical multilayer. This situation is reversed in Figure 12 where the soil configuration varies from a vertical to a horizontal multilayer. The ultimate bearing capacity was found to be the smallest for an intermediate value of the autocorrelation distance $\left(a_{x}\right.$ or $a_{y}$ ) where the failure mechanism can easily develop in the soil mass.

Tables IV and V also show the impact of the increase in $a_{y}$ or $a_{x}$ on both the skewness and the kurtosis of the PDF. As in the case of the isotropic autocorrelation distance, the PDF of the response is not far from a Gaussian one for small values of $a_{y}$ or $a_{x}$. Finally, Tables VI and VII show the effect of the increase in $a_{y}$ or $a_{x}$ on the Sobol indices $S(c)$ and $S(\varphi)$ of the two random fields. These tables show, as in the isotropic case, that the variability of the ultimate bearing capacity is mainly due to the cohesion random field, which has a Sobol index of about $71 \%$. 


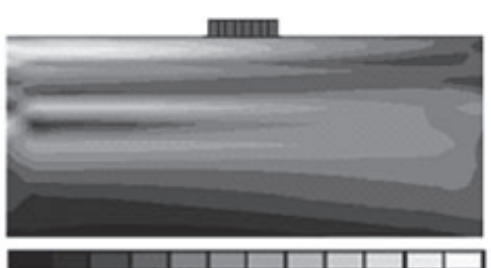

$\begin{array}{lllllllllllll}12 & 14 & 16 & 18 & 20 & 22 & 24 & 26 & 28 & 30 & 32 & 34 & 36\end{array}$ Cohesion $(\mathrm{kPa})$

(a).ax $=10 \mathrm{~m}$ and $\mathrm{ay}=0.2 \mathrm{~m}$

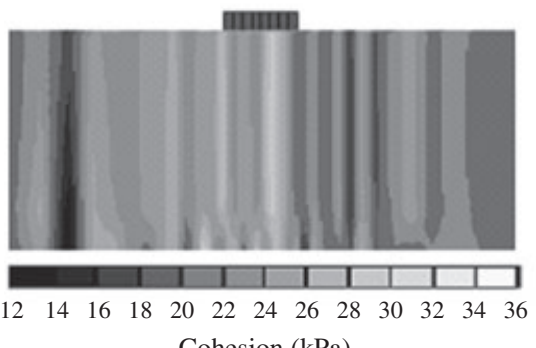

Cohesion $(\mathrm{kPa})$

(c) $\cdot a x=0.2 \mathrm{~m}$ and $\mathrm{ay}=1 \mathrm{~m}$

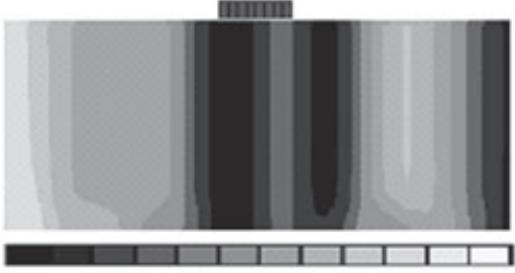

$\begin{array}{lllllllllllll}12 & 14 & 16 & 18 & 20 & 22 & 24 & 26 & 28 & 30 & 32 & 34 & 36\end{array}$ Cohesion $(\mathrm{kPa})$

(b). $a x=1 \mathrm{~m}$ and $\mathrm{ay}=10000 \mathrm{~m}$

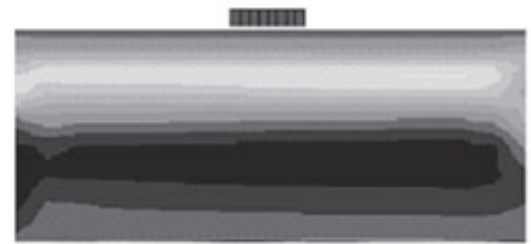

$\begin{array}{lllllllllllll}12 & 14 & 16 & 18 & 20 & 22 & 24 & 26 & 28 & 30 & 32 & 34 & 36\end{array}$ Cohesion $(\mathrm{kPa})$

(d). $a x=10000 m$ and $a y=1 m$

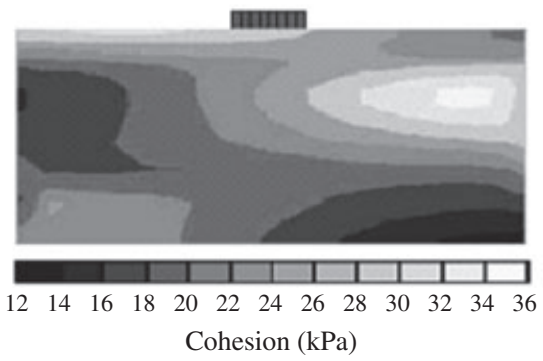

(e). $a x=10 m$ and $a y=1 m$

Figure 13. Cohesion random field for different values of the autocorrelation distance.

Table VI. Effect of the vertical autocorrelation distance $a_{\mathrm{y}}$ on the Sobol indices of the two random fields $c$ and $\varphi$.

\begin{tabular}{lcc}
\hline$a_{y}(\mathrm{~m})$ & $S(c)$ & $S(\varphi)$ \\
\hline 0.5 & 0.711 & 0.289 \\
0.8 & 0.712 & 0.287 \\
1 & 0.715 & 0.285 \\
2 & 0.713 & 0.287 \\
5 & 0.716 & 0.284 \\
8 & 0.739 & 0.260 \\
\hline
\end{tabular}

Table VII. Effect of the horizontal autocorrelation distance $\mathrm{a}_{\mathrm{x}}$ on the Sobol indices of the two random fields $c$ and $\varphi$.

\begin{tabular}{lcc}
\hline$a_{x}(\mathrm{~m})$ & $S(c)$ & $S(\varphi)$ \\
\hline 2 & 0.675 & 0.325 \\
4 & 0.706 & 0.294 \\
10 & 0.715 & 0.285 \\
20 & 0.723 & 0.276 \\
30 & 0.731 & 0.269 \\
50 & 0.730 & 0.270 \\
\hline
\end{tabular}


3.3.3. Effect of the cross-correlation coefficient. Figure 14 presents the PDFs of the ultimate bearing capacity for negatively cross-correlated $r(c, \varphi)=-0.5$ and non-correlated $r(c, \varphi)=0$ random fields when $a_{x}=10 \mathrm{~m}$ and $a_{y}=1 \mathrm{~m}$, and Table VIII presents the corresponding four statistical moments. Figure 14 and Table VIII show that the variability of the ultimate bearing capacity decreases when considering a negative correlation between the two random fields. This is because the increase of one parameter value implies a decrease in the other parameter. Thus, the total shear strength slightly varies. This leads to a reduced variation in the ultimate bearing capacity. It should be mentioned that the probabilistic mean value of the ultimate bearing capacity slightly increases when a negative correlation between the two random fields exists. Finally, the Sobol indices presented in Table IX show the same behavior detected in the previous sections.

3.3.4. Effect of the coefficients of variation of the random fields. Figure 15 presents the PDFs of the ultimate bearing capacity for three different configurations of the coefficients of variation of the random fields. Notice that for the three configurations $r(c, \varphi)=-0.5, a_{x}=10 \mathrm{~m}$, and $a_{y}=1 \mathrm{~m}$. Tables X and XI present (for the three configurations) the four statistical moments of the ultimate bearing capacity and the Sobol indices of the two fields $(c, \varphi)$. Figure 15 and Table $\mathrm{X}$ show that the variability of the ultimate bearing capacity increases when the coefficients of variation of the random fields increase; the increase being more significant for the cohesion parameter. From Table XI, one can see that an increase in the coefficient of variation of a soil parameter increases its Sobol index and, thus, its weight in the variability of the ultimate bearing capacity. This automatically reduces the contribution of the other uncertain parameter. This increase is more significant for the soil friction angle. This is because an increase by $100 \%$ in the coefficient of variation of the cohesion parameter increases its Sobol index by about 35\%, whereas increasing the coefficient of variation of the friction angle by only $50 \%$ increases its Sobol index by about $50 \%$.

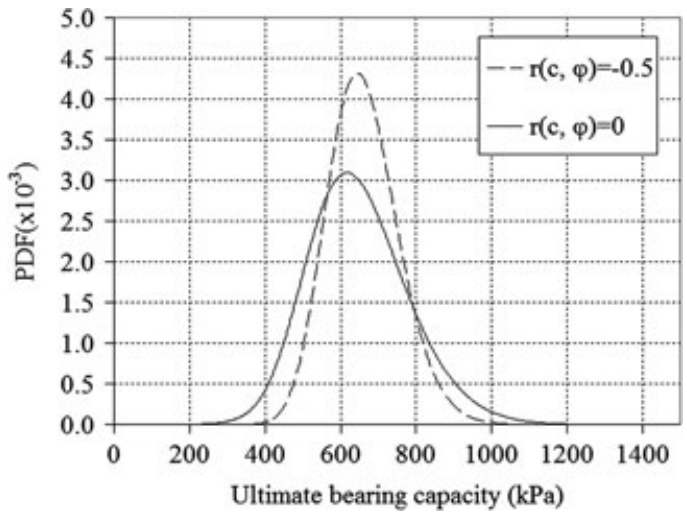

Figure 14. Influence of the cross-correlation coefficient on the PDF of the ultimate bearing capacity in the case where $a_{\mathrm{x}}=10 \mathrm{~m}$ and $a_{\mathrm{y}}=1 \mathrm{~m}$.

Table VIII. Effect of the cross-correlation coefficient between the random fields of $c$ and $\varphi$ on the statistical moments of the ultimate bearing capacity.

\begin{tabular}{lccccc}
\hline$r(c, \varphi)$ & $\mu_{q_{u t}}(\mathrm{kPa})$ & $\sigma_{q_{u t r}}(\mathrm{kPa})$ & $\mathrm{COV} \%$ & $\delta_{u}(-)$ & $\kappa_{u}(-)$ \\
\hline-0.5 & 658.2 & 93.6 & 14.22 & 0.345 & 0.201 \\
0 & 648.3 & 133.4 & 20.60 & 0.418 & 0.344 \\
\hline
\end{tabular}

Table IX. Effect of the coefficient of correlation on the Sobol indices of the two random fields $c$ and $\varphi$.

\begin{tabular}{lcc}
\hline$r(c, \varphi)$ & $S(c)$ & $S(\varphi)$ \\
\hline-0.5 & 0.715 & 0.285 \\
0 & 0.721 & 0.279 \\
\hline
\end{tabular}




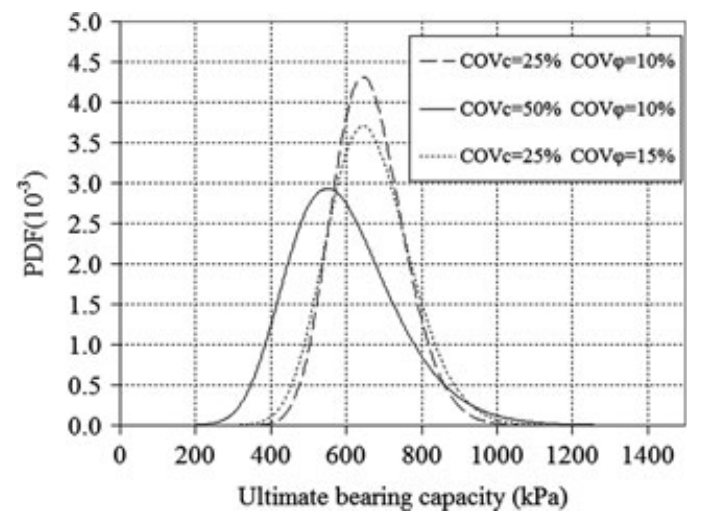

Figure 15. Effect of the coefficients of variation of the random fields on the PDF of the ultimate bearing capacity in the case where $a_{\mathrm{x}}=10 \mathrm{~m}$ and $a_{\mathrm{y}}=1 \mathrm{~m}$.

Table X. Effect of the coefficients of variation of the random fields $c$ and $\varphi$ on the statistical moments of the ultimate bearing capacity.

\begin{tabular}{llccccr}
\hline & & $\mu_{q_{u l t}}(\mathrm{kPa})$ & $\sigma_{q_{u l t}}(\mathrm{kPa})$ & $\operatorname{COV} \%$ & $\delta_{u}(-)$ & $\kappa_{u}(-)$ \\
\hline $\operatorname{COV} c=25 \%$ & $\operatorname{COV} \varphi=10 \%$ & 658.2 & 93.6 & 14.22 & 0.345 & 0.201 \\
$\operatorname{COV} c=50 \%$ & $\operatorname{CoV} \varphi=10 \%$ & 595.7 & 140.97 & 23.66 & 0.574 & 0.573 \\
$\operatorname{COV} c=25 \%$ & $\operatorname{COV} \varphi=15 \%$ & 664.2 & 108.35 & 16.31 & 0.327 & 0.185 \\
\hline
\end{tabular}

Table XI. Effect of the coefficients of variation of the random fields $c$ and $\varphi$ on the Sobol indices of the two random fields $c$ and $\varphi$.

\begin{tabular}{llcc}
\hline & & $S(c)$ & $S(\varphi)$ \\
\hline $\operatorname{COV} c=25 \%$ & $\operatorname{COV} \varphi=10 \%$ & 0.675 & 0.325 \\
$\operatorname{COV} c=50 \%$ & $\operatorname{COV} \varphi=10 \%$ & 0.915 & 0.085 \\
$\operatorname{COV} c=25 \%$ & $\operatorname{COV} \varphi=15 \%$ & 0.511 & 0.489 \\
\hline
\end{tabular}

\section{CONCLUSIONS}

A probabilistic model that considers the spatial variability of the soil properties was presented to compute the PDF of the ultimate bearing capacity of a strip footing resting on a spatially varying $(c, \varphi)$ weightless soil. The soil cohesion and friction angle were considered as two anisotropic nonGaussian cross-correlated random fields. The methodology proposed by Vořechovsky [19] was used to generate the two random fields. The SPCE methodology was employed for the probabilistic analysis. The adaptive algorithm suggested by Blatman and Sudret [18] to build up a SPCE was adopted to obtain a meta-model (i.e. an approximate analytical expression) of the ultimate bearing capacity. Finally, this meta-model was employed to perform the probabilistic analysis using Monte Carlo simulation technique.

The probabilistic numerical results were presented in the case of a weightless soil mass. This means that the present results only provide the component of the ultimate bearing capacity related to the soil cohesion. The main reason for which the soil weight was neglected is the validation of the probabilistic approach with the use of a non-expensive deterministic model that has a reasonable computation time. The numerical results have shown the interest of the SPCE methodology with respect to the classical PCE method in the case of random fields where a significant number of random variables (up to 88) were used in the analysis. The numerical results have also shown that the variability of the ultimate 
bearing capacity increases with the increase in the coefficients of variation of the random fields; the increase is more significant for the cohesion parameter. The variability of the ultimate bearing capacity is mainly due to the cohesion random field, which has a Sobol index of about $71 \%$ compared with the friction angle random field whose Sobol index is about $29 \%$. This result is logical in our case where a weightless soil was considered; the $N \gamma$ term, which is very sensitive to $\varphi$ being absent in this paper. It was also shown that an increase in the coefficient of variation of a soil parameter $(c$ or $\varphi$ ) increases its Sobol index and, thus, its weight in the variability of the system response and decreases the weight of the other parameter; the increase is more significant for the friction angle. The negative correlation between the soil shear strength parameters decreases the response variability.

With a decrease in the autocorrelation distances $\left(a_{x}\right.$ or $a_{y}$ or $\left.a_{x}=a_{y}\right)$, a less spread out (PDF) of the ultimate bearing capacity was obtained. The probabilistic mean value of the ultimate bearing capacity presents a minimum. This minimum was obtained in the isotropic case when the autocorrelation distance is nearly equal to the footing breadth $\mathrm{B}$; whereas for the anisotropic case, this minimum was obtained (for prescribed footing and soil characteristics) at a given value of the ratio between the horizontal and the vertical autocorrelation distances. Small values of the autocorrelation distances lead to small values of the skewness and kurtosis of the system response. Thus, a PDF of the system response that is not far from a Gaussian one was obtained in these cases. Finally, the obtained results show the importance of considering the spatial variability of soil properties in the probabilistic studies because some observed phenomena (such as the non-symmetrical soil failure) can not be seen when homogenous soils are considered.

\section{REFERENCES}

1. Griffiths DV, Fenton GA. Bearing capacity of spatially random soil: the undrained clay Prandtl problem revisited. Geotechnique 2001; 51:351-359.

2. Griffiths DV, Fenton GA, Manoharan N. Bearing capacity of rough rigid strip footing on cohesive soil: Probabilistic study. Journal of Geotechnical and Geoenvironmental Engineering (ASCE) 2002; 128:743-755.

3. Fenton GA, Griffiths DV. Bearing capacity prediction of spatially random c- $\varphi$ soils. Canadian Geotechnical Journal 2003; 40:54-65.

4. Popescu R, Deodatis G, Nobahar A. Effects of random heterogeneity of soil properties on bearing capacity. Probabilistic Engineering Mechanics 2005; 20:324-341.

5. Youssef Abdel Massih D. Analyse du comportement des fondations superficielles filantes par des approches fiabilistes. Ph.D. Thesis, University of Nantes, 2007; p. 267.

6. Soubra AH, Youssef Abdel Massih D, Kalfa M. Bearing capacity of foundations resting on a spatially random soil. Geocongress, ASCE, GSP N 178, New Orleans, Louisiana, USA, 9-12 March 2008; 66-73.

7. Soubra AH, Youssef Abdel Massih D. Probabilistic analysis and design at the ultimate limit state of obliquely loaded strip footings. Geotechnique 2010; 60:275-285.

8. Cho SE, Park HC. Effect of spatial variability of cross-correlated soil properties on bearing capacity of strip footing. International Journal for Numerical and Analytical Methods in Geomechanics 2010; 34:1-26.

9. Isukapalli SS, Roy A, Georgopoulos PG. Stochastic response surface methods (SRSMs) for uncertainty propagation: Application to environmental and biological systems. Risk Analysis 1998; 18:357-363.

10. Huang SP, Liang B, Phoon KK. Geotechnical probabilistic analysis by collocation-based stochastic response surface method: An Excel Add-in implementation. Georisk 2009; 3:75-86.

11. Li D, Chen Y, Lu W, Zhou C. Stochastic response surface method for reliability analysis of rock slopes involving correlated non-normal variables. Computers and Geotechnics 2011; 38:58-68.

12. Mollon G, Dias D, Soubra AH. Probabilistic analysis of pressurized tunnels against face stability using collocationbased stochastic response surface method. Journal of Geotechnical and Geoenvironmental Engineering ASCE 2011; 137:385-397.

13. Houmadi Y, Ahmed A, Soubra AH. Probabilistic analysis of a one-dimensional soil consolidation problem. Georisk 2012; 6(1):36-49.

14. Ghanem R, Spanos P. Stochastic finite elements: A spectral approach. Courier Dover Publications, $2003 ; 224$.

15. Matthies HG, Keese A. Galerkin methods for linear and nonlinear elliptic stochastic partial differential equations. Computer Methods in Applied Mechanics and Engineering 2003; 194:4521-4537.

16. Le Maître OP, Reagan M, Najm HN, Ghanem RG. A stochastic projection method for fluid flow-II. Random process. Journal of Computational Physics 2002; 181:9-44.

17. Berveiller M, Sudret B, Lemaire M. Stochastic finite elements: a non intrusive approach by regression. European Journal of Computational Mechanics 2006; 15(1-3):81-92.

18. Blatman G, Sudret B. An adaptive algorithm to build up sparse polynomial chaos expansions for stochastic finite element analysis. Probabilistic Engineering Mechanics 2010; 25:183-197. 
19. Vořechovský M. Simulation of simply cross-correlated random fields by series expansion methods. Structural Safety 2008; 30:337-363.

20. Li CC, Der Kiureghian A. Optimal discretization of random fields. Journal of Engineering Mechanics 1993; 119:1136-1154.

21. Nataf A. Détermination des distributions de probabilités dont les marges sont données. Comptes Rendus de l'Académie des Sciences 1962; 225:42-43 (in French).

22. Spanos PD, Ghanem R. Stochastic finite element expansion for random media. Journal of Engineering Mechanics 1989; 115:1035-1053.

23. Xiu D, Karniadakis GE. The Wiener-Askey polynomial chaos for stochastic differential equations. Journal of Scientific Computing 2002; 24:619-644.

24. Sudret B. Global sensitivity analysis using polynomial chaos expansion. Reliability Engineering and System Safety 2008; 93:964-979.

25. Blatman G, Sudret B. Sparse polynomial chaos expansions and adaptive stochastic finite element using a regression approach. Comptes Rendus Mécanique 2008; 336:518-523.

26. Blatman G, Sudret B. Adaptive sparse polynomial chaos expansions based on least angle regression. Journal of Computational Physics 2011; 230:2345-2367.

27. Sobol IM. Global sensitivity indices for nonlinear mathematical models and their Monte Carlo estimates. Mechanics and Computation in Simulation 2001; 55:271-280.

28. Youssef Abdel Massih D, Soubra AH, Low BK. Reliability-based analysis and design of strip foundations against bearing capacity failure. Journal of Geotechnical and Geoenvironmental Engineering ASCE 2008; 134:917-928.

29. Youssef Abdel Massih D, Soubra AH. Reliability-based analysis of strip footings using response surface methodology. Internatinal Journal of Geomechanics ASCE 2008; 8:134-143.

30. Mollon G, Dias D, Soubra AH. Probabilistic analysis of circular tunnels in homogeneous soil using response surface methodology. Journal of Geotechnical and Geoenvironmental Engineering ASCE 2009; 135:1314-1325.

31. Mollon G, Dias D, Soubra AH. Probabilistic analysis and design of circular tunnels against face stability. Internatinal Journal of Geomechanics ASCE 2009; 9:237-249.

32. Yucemen MS, Tang WH, Ang AHS. A Probabilistic Study of Safety and Design of Earth Slope. Civil Engineering Studies, Structural Research Series, Vol. 402. University of Illinois: Urbana, IL, 1973.

33. Lumb P. Safety factors and the probability distribution of soil strength. Canadian Geotechnical Journal 1970; 7:225-242.

34. El-Ramly H, Morgenstern NR, Cruden DM. Probabilistic stability analysis of a tailing dyke on presheared clay-shale. Canadian Geotechnical Journal 2003; 40:192-208.

35. Phoon KK, Kulhawy FH. Characterization of geotechnical variability. Canadian Geotechnical Journal 1999; 36:612-624.

36. Der Kiureghian A, Ke JB. The stochastic finite element method in structural reliability. Probabilistic Engineering Mechanics 1988; 3:83-91. 